\title{
Norois
}

Environnement, aménagement, société

$232 \mid 2014$

Modes de vie, modes d'habiter des aînés entre inclusion et exclusion

\section{Une enquête géo-photographique participative pour interroger les modes d'habiter des seniors tourangeaux : une proposition méthodologique}

A participatory geo-photographic survey to examine dwelling modes of seniors in Tours: a methodological proposal

\section{Béatrice Chaudet et Carine Péribois}

\section{(2) OpenEdition}

\section{Journals}

Édition électronique

URL : http://journals.openedition.org/norois/5147

DOI : $10.4000 /$ norois.5147

ISSN : $1760-8546$

Éditeur

Presses universitaires de Rennes

Édition imprimée

Date de publication : 30 octobre 2014

Pagination : 23-34

ISBN : 978-2-7535-3974-7

ISSN : 0029-182X

\section{Référence électronique}

Béatrice Chaudet et Carine Péribois, «Une enquête géo-photographique participative pour interroger les modes d'habiter des seniors tourangeaux : une proposition méthodologique », Norois [En ligne], 232 | 2014, mis en ligne le 30 octobre 2016, consulté le 19 avril 2019. URL : http:// journals.openedition.org/norois/5147 ; DOI : 10.4000/norois.5147 

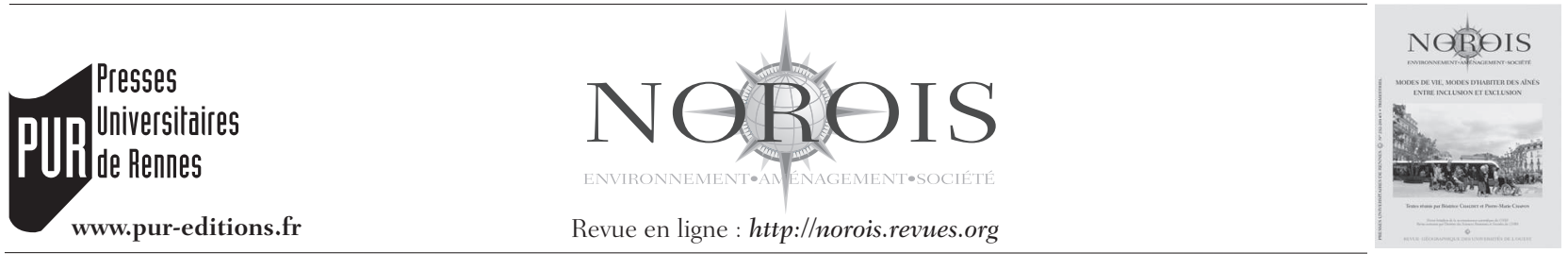

\title{
Une enquête géo-photographique participative pour interroger les modes d'habiter des seniors tourangeaux : une proposition méthodologique
}

\author{
A participatory Geo-photographic Survey to Examine Dwelling Modes of Seniors in Tours: \\ A Methodological Proposal
}

\author{
Béatrice Chaudet ${ }^{* a}$, Carine PÉribois ${ }^{b}$
}

\begin{abstract}
* Auteur correspondant
a ESO-Nantes, UMR 6590 CNRS, Institut de Géographie et d'Aménagement Régional de l’Université de Nantes, Campus du Tertre BP 81227 -44312 NANTEs cedex 3, France (beatrice.chaudet@univ-nantes.fr)

${ }^{\text {b }}$ Maison des Sciences de l'Homme Ange-Guépin, 5 allée Jacques-Berque BP 12105 - 44021 Nantes cedex 1,

France (carine.peribois@univ-nantes.fr)
\end{abstract}

Résumé : Face à la multiplication des formes d'habitats intermédiaires dédiés aux seniors autonomes et à la volonté de vieillir chez soi le plus longtemps possible tout en conservant ses pratiques socio-spatiales (Argoud, 2011), nous avons choisi d'interroger les modes de vie des personnes âgées par le biais d'une enquête géo-photographique participative. L'étude menée à Tours à l'automne 2010, dans le cadre du projet européen «Qualité de vie des seniors en Europe » et du Label « Bien Vieillir - Vivre ensemble », utilise les méthodes d'enquête des sciences sociales en s'appuyant sur des photographies produites par les seniors eux-mêmes. Images révélatrices de leur territoire de vie quotidien mais aussi des stratégies habitantes en terme de mobilité ou à l'inverse d'immobilité, les photographies apparaissent ici comme un support de parole, notamment pour évoquer les raisons d'un changement de lieu de résidence ou du maintien à domicile des personnes âgées. Cette technique d'enquête associant la photographie et le discours vise à appréhender les parcours de vie, les expériences des espaces proches du domicile. Ces photographies sont autant de traces, d’indices et de marqueurs d'un espace habité, qu’il soit imposé ou au contraire choisi. Par son approche participative ascendante, la démarche méthodologique adoptée permet de rendre compte de la diversité des expériences habitantes et d'appréhender la qualité de l'environnement résidentiel des seniors. À travers leurs regards géo-photographiques, les seniors révèlent que ce n'est pas tant la seule adaptation de l'habitat qui est à mettre en œuvre mais bien l'articulation de ce dernier avec son environnement proche. L'analyse des modes d'habiter des seniors tourangeaux, par le biais de l'enquête participative, souligne également la nécessaire adéquation entre qualité de l'environnement et capacités des individus vieillissants.

\begin{abstract}
Given the proliferation of intermediate housing forms for autonomous seniors and their wish to age at home for as long as possible, while maintaining socio-spatial practices (Argoud, 2011), we chose to examine the lifestyles of elderly people with a participatory geo-photographic survey. The study, carried out in Tours in autumn 2010, within the framework of the European project "Quality of life of seniors in Europe" and the label "Ageing Well - Living Together", uses social sciences survey methods and is based on photographs produced by the seniors themselves. As the pictures reveal of their daily life territory and also of inhabitants strategies in terms of mobility or immobility, the photographs appear here as a medium facilitating speech, in particular to discuss the reasons of the elderly for changing their place of residence or remaining at home. This survey technique combining photography and discourse seeks to cover the life courses and experiences in areas close to home. They are all traces, indices and markers of living space, imposed or otherwise chosen by the seniors. With its bottom-up participatory approach, this methodology allows us to show the diversity of inhabitants' experiences and to understand the quality of the residential environment of the elderly. Through their geo-photographic views, the seniors show that it is not only the
\end{abstract}


adaptation of housing that needs to be implemented but also the articulation of housing with its nearby environment. The analysis of dwelling modes of seniors in Tours, through the participatory survey, also highlights the necessary balance between environmental quality and the abilities of older people.

Mots clés : Qualité de l'environnement - vieillissement - mobilité - immobilité - enquête photographique participative

Keywords: Quality of environment - aging - mobility - immobility - photovoice

\section{INTRODUCTION}

"Photographiez votre habitat et son environnement proche ", telle était la consigne de notre enquête géo-photographique à laquelle une vingtaine de seniors tourangeaux a participé à l'automne 2010. La centaine de clichés ainsi obtenue a permis de comprendre, selon une approche sensible et urbaine, leurs modes de vie. Cette enquête géo-photographique repose sur les témoignages des habitants tourangeaux sur la qualité de leur environnement résidentiel. Les photographies, support du discours, permettent aux photographes enquêtés de décrire leur lieu de vie et/ou leur pratique des lieux, proches de leur domicile, et au chercheur d'appréhender la relation entre les modes d'habiter et le vieillissement de la population dans sa diversité (Membrado et Rouyer, 2013).

Le contexte démographique, marqué par la progression des effectifs de la population vieillissante, conduit les acteurs du territoire (élus, aménageurs, urbanistes, etc.) à interroger les usages de l'espace autour du domicile et son adaptation aux modes de vie des seniors. Depuis les années 2000, l'étude des modes de vie et des modes d'habiter de cette frange croissante de la population a fait l'objet de travaux de recherche principalement centrés sur l'émergence de nouvelles formes d'habitations qui leur sont dédiées (Pihet, 2003; Nowik et Thalineau, 2014; Labit et Chaland, 2012). La question de la qualité de l'environnement résidentiel et son appropriation par les personnes âgées est plus récente (Chaudet, 2012; Chapon et al., 2010; Nader, 2011). Ainsi, notre approche dépasse la simple expression du rapport à l'espace résidentiel, pour évoquer la qualité de l'environnement de leur espace de vie ou du territoire urbain de proximité (Guérin-Pace, 2003). Les entretiens réalisés se concentrent sur les pratiques spatiales quotidiennes à travers les rapports au logement, à l'espace proche du domicile, au quar- tier voire à l'ensemble de la ville. Ils révèlent ainsi les stratégies habitantes en termes de mobilité ou à l'inverse d'immobilité résidentielles et/ou quotidiennes, au regard de la qualité de l'environnement dans lequel les personnes âgées ont vécu, résident aujourd'hui ou envisagent d'habiter demain.

Face à la volonté de vieillir chez soi le plus longtemps possible en conservant ses pratiques sociospatiales, nous considérons l'habitat des personnes âgées et leur environnement proche comme un système. Celui-ci repose sur l'articulation entre les facteurs personnels (état de santé, aptitude de l'individu), les facteurs socio-économiques (revenu, condition de vie) et les facteurs environnementaux (qualité du cadre de vie, de l'aménagement de l'espace). Les relations entre ces facteurs reposent sur l'appréciation du principe de chaîne des déplacements (Sahmi et al., 2004). Cette dernière prend en compte de manière globale les potentialités de déplacement entre le domicile et les espaces ouverts au public, proches du domicile, en considérant notamment la qualité des cheminements et des modes de transports. Ces potentialités relèvent de l'adéquation entre la qualité de l'environnement, les conditions de vie et les capacités des individus vieillissants. Dans ce contexte, l'expérience et la pratique des espaces par les seniors doivent être interrogées, afin de rendre compte des perceptions et des représentations qu'ils ont de la qualité de leur milieu de vie.

L'orientation méthodologique choisie, pour analyser ces modes d'habiter, repose sur une recherche exploratoire fondée sur une enquête géo-photographique participative, que nous allons expliciter au cours de cet article. Les résultats de cette recherche mettent en exergue trois composantes complémentaires : une dimension temporelle au regard des trajectoires de vie des participants; une dimension spatiale à travers l'attachement des photographes enquêtés à leur logement, leur quartier, leur ville 
et enfin la dimension sociale au vu des solidarités visibles et invisibles exprimées par les seniors photographes interrogés. Présentées successivement, ces trois dimensions n'en sont pas moins interdépendantes, soulignant l'impact de la qualité de l'environnement proche du domicile sur le maintien durable de leur participation sociale.

\section{UN DISPOSITIF D'ENQUÊTE INSCRIT DANS UN CONTEXTE DE RECHERCHE- ACTION}

\section{Un contexte favorable à la mise en place d'une démarche participative}

L'enquête photographique a été utilisée comme méthode de recherche complémentaire au protocole d'audit urbain du Label «Bien Vieillir - Vivre Ensemble $^{1} »$, obtenu le 2 février 2010 par la Ville de Tours ${ }^{2}$. Celui-ci précise notamment les modalités de mise en place de groupes de discussion avec la population locale senior, pour identifier les atouts et les obstacles à la vie quotidienne et envisager des améliorations. La technique d'enquête vient en prolongement de ces temps d'échanges. L'obtention de ce label renouvelle les modalités de la participation citoyenne dans les quartiers de la municipalité. Fondé sur les témoignages et l'expérience vécue des personnes âgées, ce sont les stratégies habitantes mises en œuvre pour créer un milieu de vie favorable qui sont ici considérées. Cette démarche participative est également un élément constitutif du programme européen «Europe for citizens ${ }^{3}$ » qui encourage les rencontres, les échanges et les débats entre citoyens européens, notamment les seniors, par différents moyens, comme les jumelages. Les convergences méthodologiques de ces deux projets nous ont conduit à mettre en place des groupes de discussion du 20 octobre au 5 novembre 2010 .

Six groupes de discussion ont été organisés selon deux tranches d'âges (55-75 ans et 75 ans et plus)

\footnotetext{
1. Le cahier des charges du Label est largement inspiré de la démarche « Age friendly cities » (traduit «Villes amies des aînés ») soutenu par l'Organisation Mondiale de la Santé.

2. En obtenant ce Label national, la Ville de Tours s'est engagée pour 5 ans à mettre en place un plan d'actions visant à améliorer la vie quotidienne de ses seniors. La première année étant consacrée à la réalisation d'un audit urbain.

3. Le projet QLSE se positionne dans l'Action 1 « Des citoyens actifs pour l'Europe »-Mesure 1.2. "Jumelages de villes" qui soutient le développement de réseaux créés sur la base de jumelages de villes et qui consiste à tirer parti des liens établis au niveau local entre ces villes.
}

et trois secteurs géographiques de la ville: Tours Nord, Tours Centre, Tours Sud. L'appel à participation s'est fait tout d'abord largement par voie de presse via le journal local La Nouvelle République puis de manière plus ciblée au sein du tissu associatif de la ville pour compléter les groupes aux effectifs restreints. Cette recherche active de participants volontaires nous a permis notamment de toucher une population dite «silencieuse et invisible », que sont les personnes âgées aux revenus modestes, de jeunes retraités n'ayant jamais participé aux activités municipales, des néo-tourangeaux âgés de plus de 60 ans et qui ont pu prendre la parole à cette occasion (Argoud et Puijalon, 2003). Ainsi, la composition des groupes a permis de rendre compte de la diversité de la population vieillissante au sein de la ville de Tours. Lors de ces temps d'échanges, ils ont témoigné des obstacles et des éléments facilitateurs rencontrés au quotidien dans leur logement, mais aussi au cours de leurs déplacements. Autrement dit, ils ont pu s'exprimer et apprécier la qualité de vie dans leur domicile mais aussi dans l'environnement proche de celui-ci.

Dans la continuité de ces groupes de discussion thématique, nous leur avons proposé d'aller plus loin en participant à une enquête photographique. Sur 63 seniors présents aux groupes de discussion, 26 ont accepté de participer à l'enquête et de porter un regard sensible sur leur domicile, leur environnement, leurs activités, leurs déambulations dans la ville, dans leur quartier, leur rue, leur hall d'immeuble, « chez eux » (tableau 1).

L'ambition de la démarche est de rendre compte de l'appropriation du milieu de vie et des aménités urbaines par les seniors. De par l'hétérogénéité des participants, l'enquête géo-photographique vise un double objectif : saisir leurs modes de vie dans leur diversité d'une part et comprendre les éléments qui favorisent ou au contraire limitent leur participation sociale d'autre part.

\section{La collecte du corpus photographique}

La production du corpus photographique, par les seniors eux-mêmes, s'appuie sur une démarche réflexive et active des participants (Bigando, 2013). L’objectif fixé par le comité de pilotage du Label repose sur la compréhension de leurs modes de vie. Aucune indication ne précisant le nombre de pho- 


\begin{tabular}{|l|c|l|c|}
\hline $\begin{array}{l}\text { Sexe } \\
\text { Hommes } \\
\text { Femmes }\end{array}$ & \multicolumn{2}{|l|}{} & 12 \\
\hline Tranches d'âges & 16 & Dernière situation professionnelle & 14 \\
60-74 ans & 10 & Cadres et professions intellectuelles supérieures & 2 \\
75 ans et plus & & Professions intermédiaires & 7 \\
\hline Type de ménage & 10 & Employés & 7 \\
Personne seule & 14 & Sans activité professionnelle & 6 \\
Couple sans enfant & 2 & Sans réponse & 1 \\
Couple avec enfant & & Statut d'occupation du logement & 3 \\
\hline Lieu de vie & 8 & Locataires & \\
Quartiers Tours Nord & 11 & Propriétaires & 7 \\
Quartiers Tours Centre & 7 & & 19 \\
Quartiers Tours Sud & & & \\
\hline
\end{tabular}

Tableau 1 : Profils des seniors participants (Source : Enquête photo-géographique, 2010) Profiles of senior participants

tographies attendues, le nombre de clichés transmis a varié de 4 à $96^{4}$. Les participants ont eu deux mois pour prendre leurs clichés. À l'issue de cette période, le corpus comptait près de 150 photographies reflétant l'expérience habitante de 26 retraités tourangeaux. Les photos pouvaient être des prises de vue d'intérieur, d'extérieur, d'ensemble ou localisées, reflétant une perception déficitaire ou favorable de leur mode de vie au quotidien. La composition des photographies produites est étonnante : elles donnent à voir l'espace habité dans sa singularité et sa diversité et une mise en scène de leur quotidien riche en enseignements. En effet, les photographies présentent à la fois la sphère privée du domicile permettant de visualiser la pièce de vie principale, les espaces de liaison entre le domicile et l'espace public caractérisant la qualité des aménagements d'un hall d'immeuble, des voies d'accès aux pavillons d'un lotissement. D'autres clichés témoignent des espaces proches du domicile montrant aussi bien des lieux dédiés aux seniors, à l'image du club de belote, que les commerces de proximité ouverts au public comme la pharmacie ou la boulangerie ou encore des lieux de rencontres, de promenades tels que les parcs ou les marchés. Ce corpus, dans sa diversité, révèle indéniablement une prise de conscience des situations d'entraves ou de facilitateurs à l'autonomie, la convivialité et la sécurisation du milieu de vie.

La taille du corpus photographique de chaque participant importe peu, dans la mesure où l'existence

4. Dans le respect du droit à l'image, les seniors étaient munis d'autorisations à faire signer le cas échéant aux personnes présentes sur les clichés. d'un cliché - support de l'entretien - rend possible la discussion entre le chercheur et le senior photographe. En effet, la photographie seule ne présente qu'un intérêt limité si le contexte dans lequel le cliché a été pris ou sa signification pour la personne n'est pas explicité. À cette occasion, les seniors ont accepté de lever le voile sur leur manière d'habiter à Tours et de mettre des mots sur ces clichés.

La méthode de l'enquête photographique, issue du courant de l'anthropologie et de la sociologie visuelle nord-américaine, présente l'intérêt majeur de faire parler des individus à partir d'un corpus de clichés photographiques qu'ils ont eux-mêmes produit (Collier, 1967). Ici, la photographie est utilisée comme support d'investigation pour mieux comprendre les modes d'habiter des seniors. Le rôle de l'image ne se limite pas à l'illustration d'un contexte, il est source de données que le chercheur va interpréter. C'est aussi un formidable support de l'entretien de recherche. Cette entrée facilite l'expression des participants qui ne s'adressent plus en face à face avec le chercheur, mais témoignent d'une pratique photographique. De par sa fonction médiatrice, la photographie modifie les rapports entre enquêtés et enquêteurs. Elle tend à inverser les rôles : le photographe enquêté guidant le chercheur enquêteur dans l'interprétation des clichés (Hatt, 2011). L'entretien, pour le senior, consiste à expliciter et justifier les photographies produites afin de sélectionner «la » photo qui lui semble la plus parlante pour évoquer l'habitat senior et son environnement proche. Au-delà de la photographie choisie, l'objectif du chercheur repose sur la com- 
préhension des éléments photographiés et le sens que l'auteur lui attribue. En multipliant les auteurs de ces images, le chercheur accède à une variété de points de vue qui lui permettent de confronter les représentations de l'habitat et de son environnement proche. Dès lors, les clichés qui sont donnés à voir et explicités lors de l'entretien mettent en exergue les pratiques de l'espace habité et les logiques individuelles ou collectives à l'œuvre.

\section{La photographie, support de l'entretien individuel}

Tous les participants ont été conviés individuellement à un entretien enregistré, sur la base du corpus photographique réalisé : ce dernier servant de support à l'entretien qui se déroule en trois temps. D'emblée, la personne est invitée à raconter chacun de ses clichés. Cette première lecture du corpus permet d'accéder aux différents registres des modes d'habiter (Cailly et Dodier, 2007). Cette entrée en matière facilite l'échange et permet au chercheur de s'imprégner des lieux de vie de l'enquêté. La photographie, par la mise en scène de l'habiter qu'elle propose, contribue à la compréhension de ses pratiques sociales et spatiales.

Le chercheur invite ensuite le photographe à trier son corpus photographique selon ses propres critères. L'absence d'indication sur la grille de lecture à appliquer aux photographies rend les enquêtés perplexes, laissant quelques minutes de silence et d'inaction avant de proposer un tri. Cette catégorisation impose au photographe un premier niveau de réflexion induit par le dispositif méthodologique. Le tri opéré varie selon les participants : certains distinguent les prises de vue intérieures et extérieures, d'autres optent pour une sélection esthétique ou opèrent un tri selon le moment de la prise de vue, d'autres enfin distinguent les photos selon la présence humaine ou non. La justification des catégories proposées et des photographies qui les constituent fait émerger un premier système de représentations des modes d'habiter. Par exemple, l'un des participants a sélectionné quelques photographies dans son album de famille. Le rôle du chercheur est alors de comprendre les changements qui se sont opérés depuis la prise de vue choisie. L'auteur des photos en question (Senior-Photographe SP17) précise que l'une des images présente le temps de la vie de couple qui n'est plus d'actualité dans la salle à manger d'une maison, dont il était propriétaire et qu'il n'habite plus aujourd'hui (figure 1). Si la photographie ne présente qu'un fragment de la réalité à un instant donné, elle rend compte d'une représentation d'un mode de vie inscrit dans le temps et dans l'espace.

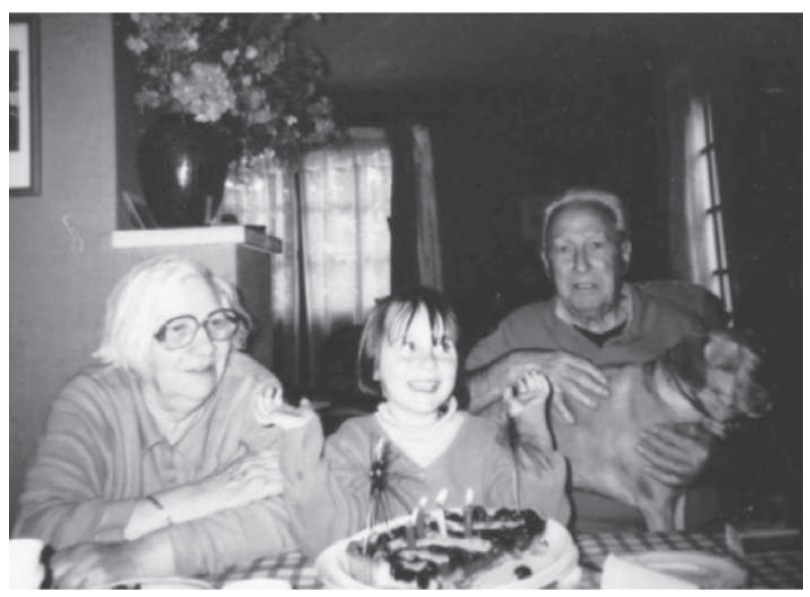

Figure 1: La photographie fige dans le temps une vie passée (Source : SP17, 2010)

The photography freezes past life in time

Le troisième temps de l'entretien consiste à sélectionner et justifier cinq photographies qui répondent au thème de l'exposition « L'habitat et son environnement proche pensé pour et par les seniors ». L'opération se répète à l'identique pour retenir trois clichés, puis «la » photographie qui fera l'objet d'une exposition grand public. Chacune de ces étapes du dispositif méthodologique conduit la personne à expliciter plus en détail les photographies qu'il choisit. Au fur et à mesure des lectures successives du corpus photographique pour sélectionner « la » photo à exposer, elle dévoile alors une série d'informations plus sensibles, relevant de son histoire personnelle et/ou familiale, de sa trajectoire résidentielle, de l'évolution de son état de santé ou celui de ses proches. La photographie apparaît ici comme un support de parole, mettant en exergue les raisons d'un changement passé ou futur de lieu de résidence, ou encore de la volonté de rester chez soi. Ainsi, à partir des clichés produits et choisis, ce sont les stratégies résidentielles et les stratégies d'appropriation de l'espace proche du domicile qui sont progressivement évoquées. 


\section{UNE LECTURE TRIDIMENSIONNELLE DES PRATIQUES SOCIO-SPATIALES DES SENIORS TOURANGEAUX}

\section{La dimension temporelle du domicile et son environnement $v$ s trajectoires de vie}

L'analyse du corpus photographique repose non seulement sur ce qui est donné à voir, mais également sur ce qui est donné à entendre. Ainsi, les « images habitées [permettent de] voir à travers les images l'intention du photographe et de saisir objectivement une réalité de ce qui fait un quotidien » (Bonnin, 2006). 20 photographes parmi les 26 ont produit des photographies prises depuis la fenêtre de leur domicile pour exprimer leur quotidien. Les discours recueillis autour de ces clichés montrent que la vue depuis son domicile rythme ce quotidien. L'un des clichés proposés présente une fenêtre fermée avec des rideaux derrière lesquels on aperçoit le passage des éboueurs. L'auteur du cliché (SP8) nous précise que « chaque jour le facteur passe en vélo vers 11 heures, mais là c'est le passage du camion des éboueurs on est un mardi, ça veut dire qu'il est bientôt 10 heures » (figure 2).

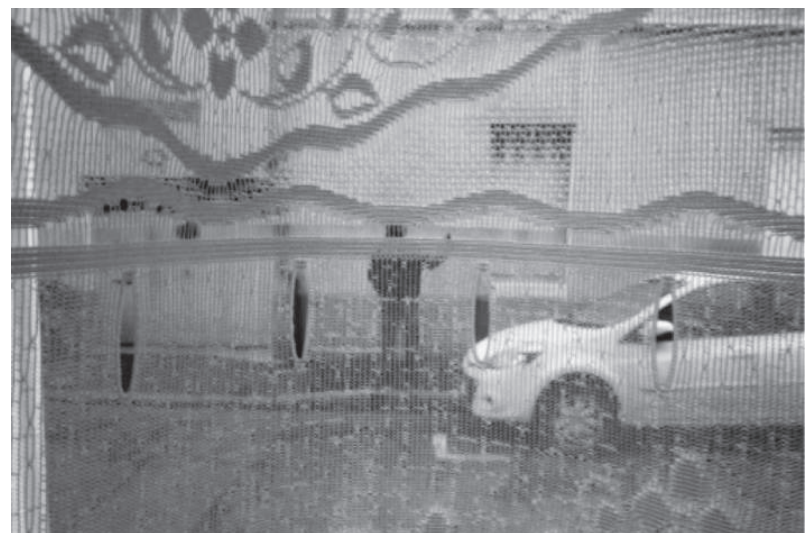

Figure 2 : Vue depuis la fenêtre et temps du quotidien (Source : SP8, 2010)

View from the window and everyday time

D'autres évoquent les changements de la vue sur les toits de la ville au rythme des saisons. Un tiers des seniors photographes interrogés partage ce point de vue. Certains ont sélectionné des séries de clichés présentant successivement les toits de la ville en hiver sous la neige ou au printemps avec le retour de la végétation (SP10, SP1). D'autres ont décrit la luminosité du domicile à différents moments de la journée, le moment du lever ou le coucher du soleil (SP11, SP5) ou encore les cris des enfants dans la cours de l'école, située en contrebas de leur appartement (SP14). La fenêtre, interface entre le dedans et le dehors, entre l'espace privé et public, rend compte des éléments qui rythment le quotidien à travers ce qui est donné à voir, à entendre depuis chez soi.

Seulement trois d'entre eux ont retenu les prises de vue de l'intérieur du logement dont deux de leur propre domicile. Force est de constater que cette catégorie de photographies nous permet d'entrer dans l'intimité de la personne. Dès lors, la photographie du domicile devient non seulement la base de l'expression d'un mode de vie présent, mais aussi le support d'un discours précisant les différences avec le logement précédent ou celui à venir, marquant ainsi les parcours résidentiels des seniors. Une personne (SP1) a retenu la photo de la pièce principale de son logement, dans laquelle « tout est complet... dans la bibliothèque là (à droite) vous voyez ma collection de coquillages de mes voyages, là j'aime les livres, le landau que j'ai acheté à la brocante pour mettre mes fleurs [...] il manque juste ma clarinette mais là ça ferait trop, j'avais pas la place ». La description de la photographie est l'occasion pour lui de retracer les grands évènements de sa vie passée et présente (figure 3). Il souligne aussi l'attachement à son domicile inchangé depuis son arrivée à Tours, son histoire personnelle et le confort acquis dans son logement.

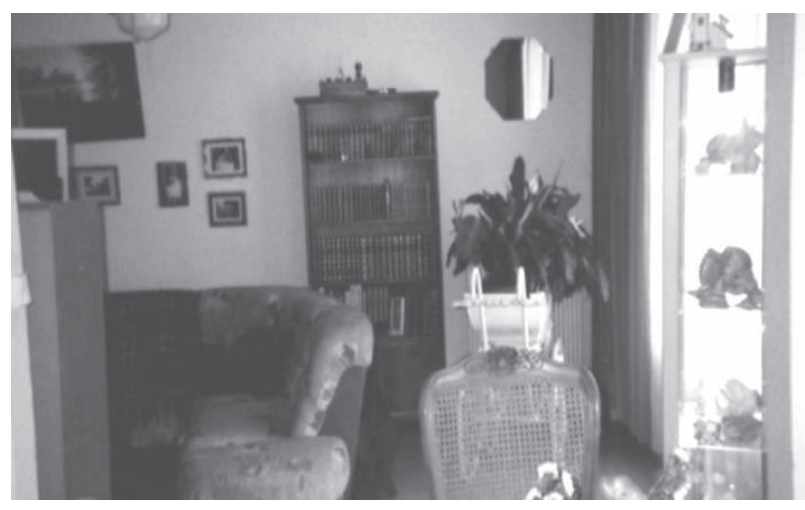

Figure 3 : La photographie, témoin d'une vie passée (Source : SP1) The photography as the witness of a past life

Un autre (SP23) retient, quant à lui, la photographie d'un couple dans son salon, caractérisant le confort, le bien-être à la retraite et la grande tran- 
quillité du logement, son accessibilité et le repos, notamment depuis que l'un des deux rencontre des difficultés de santé lui imposant un déplacement régulier vers l'hôpital. Autre sélection, celle d'un senior qui souligne lui aussi les conséquences de l'évolution de l'état de santé de son conjoint et son incidence sur leur trajectoire de vie et résidentielle commune. Enfin, en sélectionnant une photo de famille prise dans la pièce principale de sa maison, l'un des participants évoque le douloureux déménagement suite à l'évolution de la maladie d'Alzheimer de son épouse, entraînant le changement de domicile vers une institution pour elle et vers un logement plus petit pour lui (SP17). La mobilité résidentielle imposée par l'état de santé de l'un et la taille d'un logement familial inadapté pour l'autre souligne implicitement l'adaptation du domicile au vieillissement de ses propriétaires. L'un des individus confirme cette difficile transition entre le pavillon individuel et l'appartement en centre-ville. Il précise alors que "changer de logement c'est aussi changer de mode de vie » (SP19). De même, une personne (SP3) interroge son propre vieillissement en retenant la photographie de sa baignoire, confortable dans le passé, encore accessible aujourd'hui, mais d'un usage vraisemblablement plus difficile à l'avenir, ce qui pose la question de l'adaptation future de l'intérieur de son logement.

La diversité des images et des discours recueillis met ainsi l'accent sur les stratégies habitantes en terme de mobilité ou à l'inverse d'immobilité. Les mobilités résidentielles, qu'elles soient choisies ou imposées, visent à maintenir l'adéquation entre les facteurs personnels, environnementaux et socio-économiques en vue de conserver, voire d'accroitre son autonomie. Quel que soit l'angle de prise de vue choisi (intérieur ou extérieur vu de l'intérieur), les clichés soulignent l'articulation entre le domicile et son environnement, faits de mobilités et d'ancrages territoriaux.

\section{La dimension spatiale des clichés, révélatrice d'un ancrage territorial}

L'ancrage au territoire apparaît sur les clichés pris hors du domicile. De fait, la majorité des participants (24 sur 26) a fait le choix de photographier l'environnement proche de son domicile. Ces derniers présentent les évènements ponctuels auxquels ils ont assisté (SP21), les lieux qu'ils fréquentent avec leurs proches à l'image des parcs et jardins (SP18, SP20, SP24), des rives de la Loire (SP13), du Cher (SP19, SP26), les animations auxquelles ils participent régulièrement : c'est le cas du marché tous les dimanches ou du marché aux fleurs deux fois par semaine, dont l'auteur (SP15) souligne «l'intemporalité et la beauté [...] toute l'année ». Ce registre de photographies caractérise la mobilité quotidienne des retraités tourangeaux, la diversité des lieux fréquentés, la place prédominante accordée à la nature en ville (figure 4).

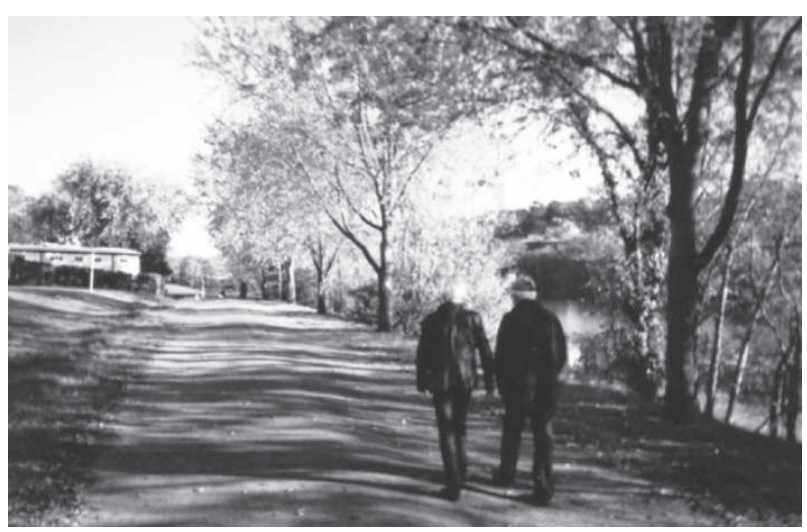

Figure 4 : Les rives du Cher offrent la nature près de chez soi (Source : SP26, 2010)

The banks of the Cher River bring nature close to home

Ces lieux photographiés tendent à montrer la place prédominante accordée à la marche quotidienne. Les participants ont capturé des images révélatrices de leur territoire de vie quotidien vécu et perçu. Chacun d'entre eux signale la qualité de l'environnement à Tours, la beauté des paysages, la proximité des lieux de promenade ou la présence de commerces et de services proches du domicile. L'adéquation entre l'environnement et l'état de santé de la personne influence les mobilités des seniors. Certains soulignent que "la pratique de la marche tous les jours, [...] fait partie de [leur] ordonnance » (SP4, SP5) et participe au maintien de leur autonomie dans leur logement et au sein de leur quartier. Or, en fonction de l'aménagement des espaces proches du domicile, certains limitent leurs déplacements quotidiens, compte tenu de leur avancée en âge et de ses effets : "Autrefois on marchait plus, aujourd'hui c'est moins facile »(SP13). D'autres encore insistent sur les obstacles au maintien de la 
mobilité qui relèvent en partie des aménagements urbains inadéquats, tels que l'absence de bancs ou des bancs disposés trop loin d'un escalier (SP4, SP5), la présence de poubelles sur les trottoirs (SP6, SP7), ou encore la qualité de la voirie (figure 5) : "les feuilles, les bosses et les fissures sur les trottoirs sont dangereuses » (SP22, SP9).

Les seniors témoignent ainsi des difficultés rencontrées au quotidien en termes d'aménagement

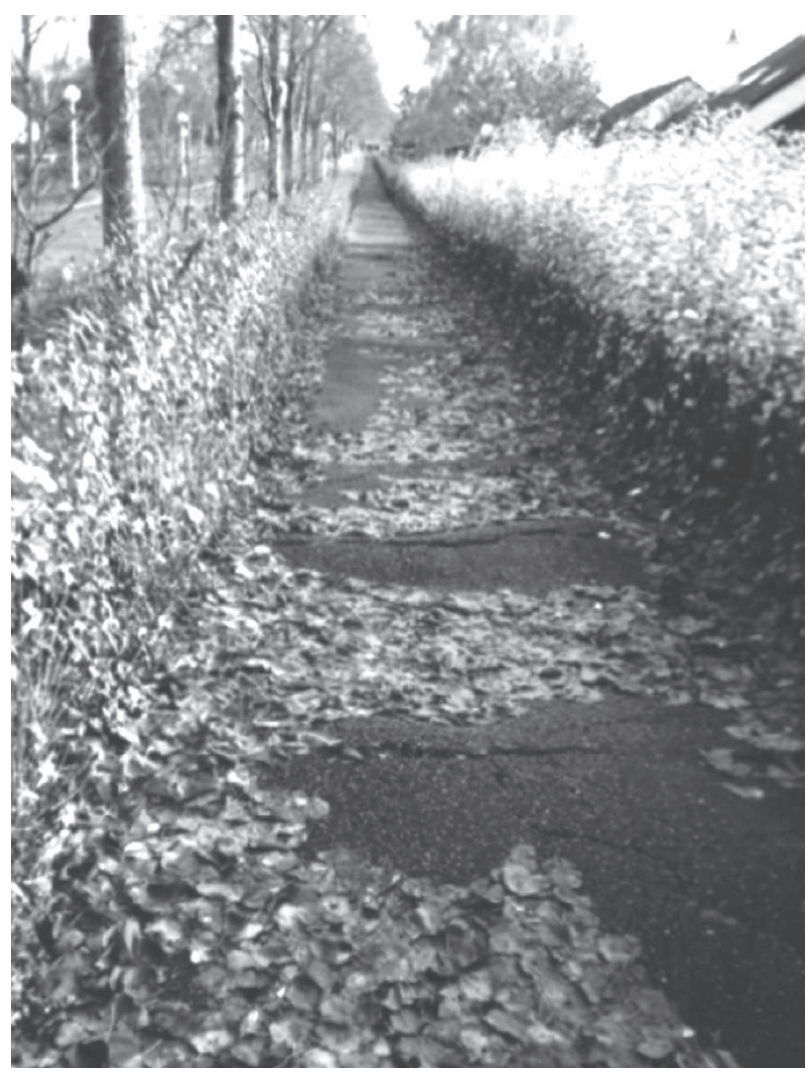

Figure 5 : La qualité de la voirie, un obstacle à la mobilité (Source : SP22, 2010)

The quality of roads, an obstacle to mobility urbain ou de capacités physiques. Certains auteurs précisent que ces dernières influencent leurs déplacements, leurs activités sociales... L'un d'entre eux, par exemple, a sélectionné la photo de vespasiennes, pour souligner l'importance de connaître l'emplacement des toilettes publiques, lorsqu'il part faire sa balade quotidienne (SP20). D'autres confirment que leur connaissance du quartier leur permet de gérer les temps de marche, les lieux de pause, l'appui sur la rambarde de l'escalier... : autant de repères, de points d'ancrages nécessaires au maintien de leur autonomie dans leur quartier (figure 6).

Ainsi, ils mettent en place de réelles stratégies à l'égard de leur habitat et de leur environnement proche. Ces stratégies habitantes soulignent, s'il en est besoin, les capacités d'adaptation des populations vieillissantes rendues possible par leur connaissance fine du territoire et de ses aménagements. Les photographies sont des marqueurs de leur mobilité. La diversité des profils des personnes concernées par ces mobilités quotidiennes hors du domicile nous conduisent à distinguer les mobilités des hommes ou femmes seul(e)s ayant un réseau social important (SP13, SP8), des couples dont la mobilité de l'un est limitée par son état de santé (SP4, SP5, SP6, SP7) et celles des seniors en situation de fragilité économique et/ou sociale (SP2, $\mathrm{SP} 3)$. Les déplacements réguliers dans la ville sont ainsi conditionnés par leur autonomie et traduisent des enjeux importants en terme d'offre d'espaces de qualité, relevant à la fois de la diversité des modes de vie et de la localisation résidentielle.

Certains privilégient les sorties, la marche, les activités sociales, tandis que d'autres moins mobiles accordent une place considérable à la vue depuis
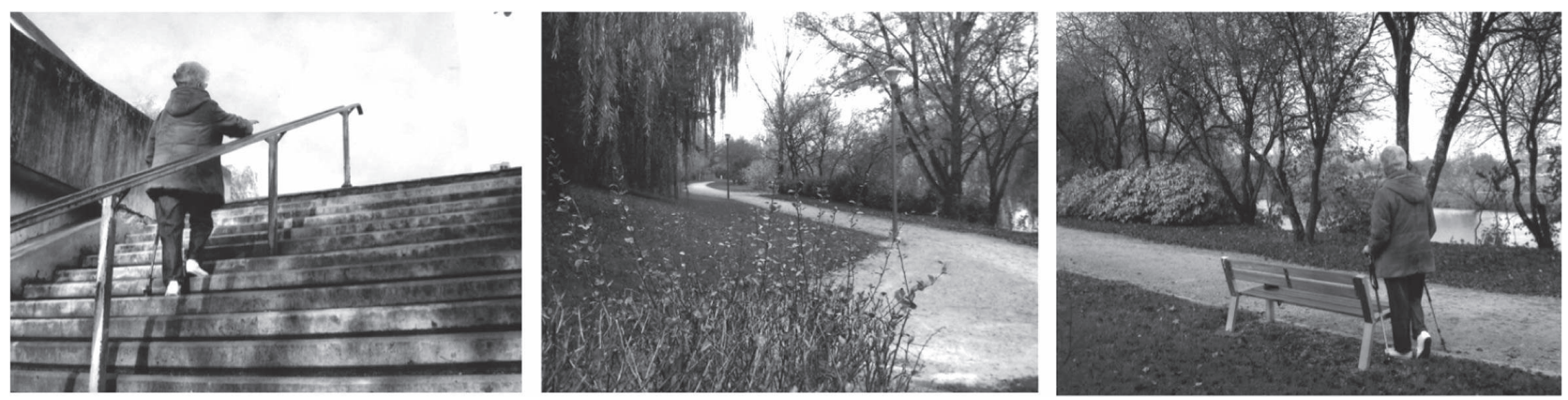

Figure 6 : Se déplacer autour du domicile, c'est d'abord anticiper sa mobilité (Source : SP4 et SP5, 2010) Moving around the house, it is first to anticipate the mobility 
leur domicile : "Tous les matins, je vérifie que le lac est bien là " (SP1) - "J'aime observer la luminosité sur ce toit depuis la [seule] fenêtre de chez moi » (SP2) - "J'ai entendu les cris des enfants et j'ai vu ces ballons devant ma fenêtre alors j'ai vu que c'était la fête dans la cours de l'école» (SP14). Ici, la mobilité est rêvée, imaginée ou vécue par procuration. Un certain nombre de photographies évoque ainsi une mobilité ancrée à la fois dans le territoire mais aussi dans leur mémoire (par exemple, un parcours de promenade effectué auparavant régulièrement dont on se souvient dans les moindres détails) : ancrages territoriaux et temporels se superposent alors. Ces registres de photos sont également des révélateurs de sons, caractéristiques du quotidien que les individus perçoivent depuis leur balcon ou leur salon. L'image introduit alors l'expression sensible de ce quotidien : parler d'une photographie amène à évoquer les paysages sonores, visuels ou olfactifs (Martouzet, 2014). Autant d'ambiances urbaines localisées auxquelles ils semblent attachés, parce qu'elles rythment leur quotidien et renouvellent les formes de mobilités.

Les clichés soulignent un attachement au lieu de vie, caractérisé par des repères liés à l'identité, l'histoire personnelle ou par des repères extérieurs, facilitant les déplacements quotidiens. Cet ancrage au territoire est en partie lié à la qualité de l'environnement qu'il soit favorable au maintien de son autonomie ou au contraire qu'il la limite. Dès lors, les solidarités à l'œuvre sont susceptibles de palier les difficultés rencontrées.

\section{La dimension sociale : solidarités - sociabilités visibles et invisibles}

D'emblée, on observe un certain équilibre entre les photos marquées par la présence humaine comme les photos de famille, de retrouvailles et celles où l'on devine l'activité sociale des seniors : certains ont choisi de photographier les membres de leur club de belote (figure 7), d'autres les espaces publics favorables à la rencontre, tels que les commerces de proximité - pharmacie, boulangerie, marché (SP12, SP15) ou encore le hall de leur immeuble (SP25).

Ainsi, le support photographique laisse apparaître des sociabilités familiales ou amicales non visibles. L'un d'entre eux a choisi de sélectionner la photo d'un jardin public dans lequel il se rend avec ses

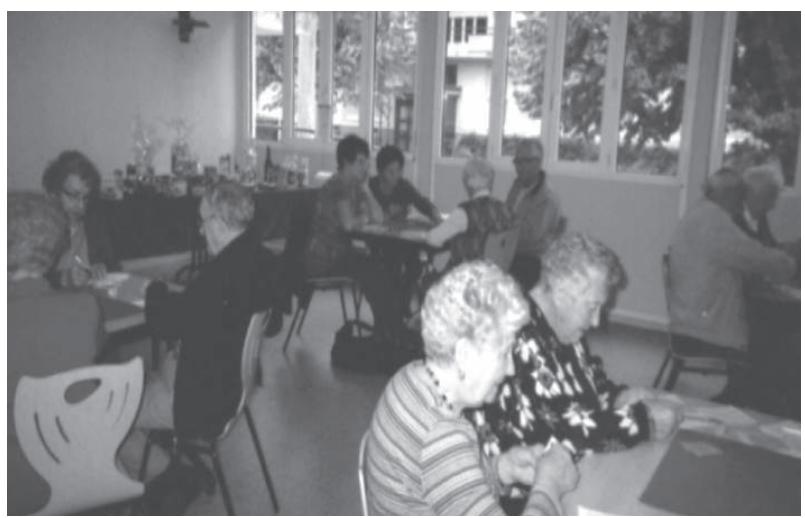

Figure 7 : Le club de belote, lieu de sociabilités (Source : SP21, 2010)

The Belote club, a place of sociability

petits-enfants, non présents sur le cliché (SP18); d'autres ont retenu les clichés d'espaces publics où ils se baladent régulièrement (SP13). D'autres encore ont sélectionné la photo d'un coucher de soleil, en référence aux photos envoyées régulièrement aux proches, amis ou famille (SP11). Les liens sociaux implicitement présents sur les photographies retenues, mais n'affichant aucune présence humaine, sont au moment de l'entretien explicités par les auteurs. On recense alors un certain nombre de photographies mettant en lumière des solidarités invisibles. Le registre des solidarités familiales, quant à lui, apparaît sous plusieurs formes : celui du moment partagé avec ses petits enfants "j'emmène ma p'tite fille au parc » (SP18), ou encore celui d'une réunion de famille pour l'anniversaire de l'arrièrepetite-fille, en présence du chien (SP16). Si la première photographie relève des solidarités invisibles, la seconde met en scène un moment de vie intergénérationnel.

Par ailleurs, quelques-uns ont opté pour des clichés soulignant une sociabilité indirecte, au sens d'attention portée aux autres en société. Par exemple, une personne propose une lecture du hall de son immeuble où la hauteur des boîtes aux lettres est susceptible de poser des difficultés à leurs propriétaires. Ce n'est pas le cas de l'auteur mais il tient à rapporter ce fait relaté à plusieurs reprises par les résidents (SP27). D'autres encore soulignent la mixité sociale de leur immeuble: "Je me fonds dans la masse, j'habite un quartier où il y a des tours, des maisons individuelles. J'habite pas un logement stigmatisant un logement seniors » (SP10). 
Le registre des sociabilités visibles est plus rare. Il repose sur la mise en scène de ces espaces collectifs où le lien social est promu. C'est le cas d'un photographe (SP8) qui évoque un épisode émouvant de « retrouvailles ", symbolisant une sociabilité retrouvée (figure 8). L'auteur du cliché nous raconte que la femme photographiée est une ancienne institutrice qui accompagnait son mari (ancien garde champêtre) pour une manifestation de quartier et retrouve à cette occasion une ancienne élève. Au-delà de la description de la photo, l'auteur du cliché tient à préciser que les métiers de garde champêtre comme celui d'institutrice occupent une place toute particulière en termes de sociabilités au sein d'un quartier.

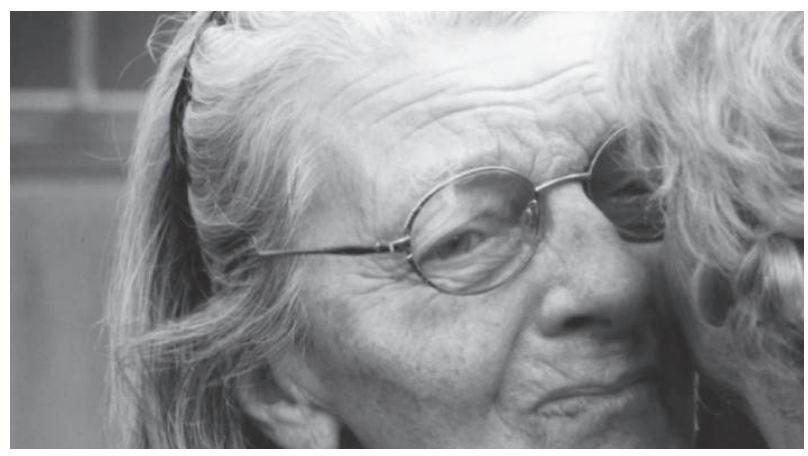

Figure 8 : La photographie, symbole de sociabilités retrouvées (Source : SP8, 2010)

The photography as a symbol of found social connections

Le dispositif méthodologique adopté a permis de montrer qu'un grand nombre de seniors a photographié les moments ou les moyens d'entrer en contact avec l'autre. Les clichés d'animaux domestiques, de plantes sur le balcon, des travaux en bas de l'immeuble sont autant d'images qui renvoient aux sujets de discussion qu'ils abordent avec la famille, les voisins ou les commerçants de proximité. D'autres ont préféré photographier les activités collectives extérieures menées par les voisins comme l'entretien des jardins partagés, du compost collectif. D’autres encore ont souhaité souligner la qualité de la gestion des espaces communs entre le domicile et la voirie (le hall d'immeuble, le parking...). Toutes ces photographies rendent compte ainsi des effets de la qualité de l'environnement social et spatial sur la capacité à participer à la vie de quartier, de la cité. Dans ce sens, une personne (SP14), surprise de voir passer un ballon sur son balcon, s'est déplacée pour assister à distance et de manière indirecte au lâcher de ballons dans la cour d'une école, en bas de chez elle. Expérience "partagée » qu'elle a souhaité immortaliser en prenant une photo (figure 9).

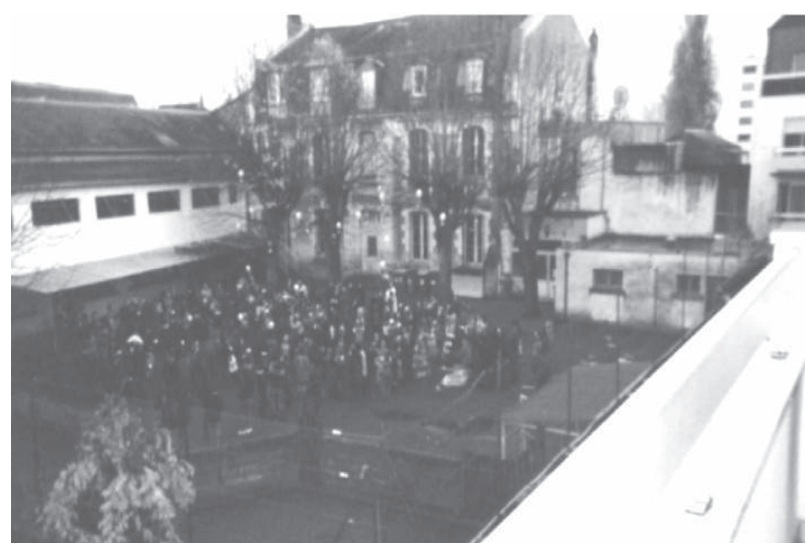

Figure 9 : La participation sociale comme indicateur du mode d'habiter (Source : SP14, 2010)

Social participation as an indicator of way of living

Si les trajectoires de vie des photographes sont fréquemment exprimées au cours de cette enquête géo-photographique, ce sont les phases de transitions résidentielles qui semblent davantage les préoccuper. En effet, le changement de domicile reste un obstacle à franchir pour la majorité d'entre eux. Le maintien à domicile dans son quartier est le cœur de leur discours. Les enjeux du changement de domicile et l'alternative vers un habitat dédié aux seniors sont rarement évoqués, voire inexistants. Le présent prime sur un futur qu'ils se refusent pour beaucoup à envisager en-dehors de leur logement, de leur quartier. Nous l'avons vu et entendu, les effets du vieillissement biologique imposent d'aménager les espaces urbains et les espaces du domicile pour permettre aux tourangeaux de maintenir voire d'accroître leur autonomie, leur mobilité. Ce sont aussi les attentes en termes d'adaptation du domicile, de liens sociaux, qui relèvent de la promotion de solidarités entre voisins et habitants du quartier, qui sont mises en avant. 


\section{Discussion}

\section{L'enquête géo-photographique, support de l'expression du mode d'habiter ordinaire}

La production de ce travail photographique et le recueil des discours associés ont ainsi permis de mettre en avant trois dimensions des modes d'habiter des seniors tourangeaux. La dimension temporelle qui apparaît au regard de leurs trajectoires de vie, la dimension spatiale à travers l'ancrage aux territoires de proximité du domicile des participants et la dimension sociale, révélant entre autres les solidarités visibles et invisibles à l'œuvre au quotidien. L'enquête photographique apparaît ici comme un moyen de faire émerger le vécu d'une population hétérogène. Les clichés laissent entrevoir des modes d'habiter ordinaires, faisant état de trajectoires personnelles et/ou collectives, inscrites dans le temps et l'espace. Chacun d'entre eux a un mode d'habiter qu'il a choisi d'exposer à travers un cliché, donnant à voir son « chez soi » selon un angle de vue singulier : intérieur, extérieur, de l'intérieur sur l'extérieur. Dans cette interprétation de l'habitat et de son environnement, la photographie prend sens à la lumière du discours des seniors qui renvoie au contexte personnel, social et spatial de l'individu.

L'approche tridimensionnelle de cette enquête géo-photographique a permis au chercheur d'observer les logements et leur environnement à partir d'un angle de vue original. Les productions photographiques commentées par les seniors eux-mêmes ont éclairé le chercheur sur leurs modes de vie et d'habiter et ont servi de supports de discussion concernant leurs activités quotidiennes, leurs lieux de résidence, leurs mobilités ou leurs immobilités, contribuant à la compréhension de leur qualité de vie. Autrement dit, elles ont permis d'observer et de retracer leur vécu mais aussi de comprendre leurs relations avec l'environnement proche du domicile et leurs rapports à la société.

\section{Valorisation de la "parole des vieux" ${ }^{5}$ " un temps d'échanges enquêteurs- enquêtés}

Le temps de la restitution aux participants mais aussi aux Tourangeaux dans leur ensemble a été rendu possible grâce à l'inscription de l'enquête photographique dans le projet européen QLSE et le Label «Bien Vieillir - Vivre ensemble ». En effet, le programme «Europe for citizens » prévoyait l'organisation d'une rencontre thématique par chaque ville partenaire. La Ville de Tours a accueilli la $4^{\mathrm{e}}$ rencontre européenne, du 20 au 22 janvier 2011, sur le thème de « l'habitat Pour et Par les seniors », en marge de laquelle s'est déroulée une exposition, présentant les clichés sélectionnés. Afin de valoriser ce travail participatif et sensibiliser la population aux modes d'habiter des seniors, l'exposition a ouvert ses portes une semaine avant la tenue de la rencontre européenne et a été prolongée jusqu'au 29 janvier 2011 dans le Péristyle de l'Hôtel de Ville de Tours. Par ailleurs, le Label «Bien vieillir - Vivre ensemble » préconise aux acteurs publics de prendre en compte la parole des personnes âgées. Dès lors, la dimension participative de l'enquête photographique a contribué à l'audit urbain de la Ville de Tours et permis une restitution aux participants des groupes de discussion, dont certains ont prolongé leur implication à travers l'enquête. L'exposition a été repensée pour être itinérante et permettre ainsi de présenter la démarche de l'enquête et la production photographique, notamment dans les mairies annexes et les foyers logements de la Ville de Tours.

\section{Bibliographie}

Argoud D., Puijalon B., 2003. Enjeux et limites d'une prise en compte de la parole des vieux, Gérontologie et société, 2003/3 n 106, p. 23-39.

Argoud D., 2011 . De l'hébergement à l'habitat : une évolution ambiguë, Gérontologie et société, 2011/1 n 136, p. 13-27.

Bigando E., 2013. De l'usage de la photo elicitation interview pour appréhender les paysages du quotidien : retour sur une méthode productrice d'une réflexivité habitante, Cybergeo: European Journal of Geography [En ligne], Politique, Culture, Représentations, document 645, mis en ligne le 17 mai 2013, URL: [http://cybergeo.revues.org/25919].

Bonnin P., 2006. Images habitées, photographies et spatialité, Paris, Creaphis, coll. «Images photo », 271 p.

5. En référence au titre de l'article d'Argoud et Puijalon (2003). 
Cailly L., Dodier R., 2007. La diversité des modes d'habiter des espaces périurbains dans les villes intermédiaires : différenciations sociales, démographiques et de genre, Norois, $n^{\circ} 205$, p. 67-80.

Chaudet B., 2012. Les territoires du « Bien vieillir » au prisme de la mobilité quotidienne des personnes âgées, in VIRIOT Durandal J.-P., Pinet C., Chapon P.-M. (dir.), Les défis territorianx face au vieillissement, Paris, La documentation Française, p. 17-30.

Chapon P.-M., Beuret C., Bolomier C., Choisy P., ZamberNARDI S., 2010. Cartes mentales et représentations spatiales de résidants en MARPA : un outil d'aide à l'implantation de nouvelles structures d'hébergement?, Norois, n² 216 , p. 57-66.

Collier J., 1967. Visual Anthropology: Photography as a Research Method, New York, Holt/Rinehart and Winston, 266 p.

Guérin-Pace F., 2003. Vers une typologie des territoires urbains de proximité, L'Espace Géographique, n 4, p. 333-345.

Натт E., 2011. Requalifier les stations touristiques contemporaines: une approche des espaces publics. Application à Gourette et Seignosse-Océan. Thèse de doctorat en aménagement et urbanisme, Université de Pau et des Pays de l'Adour, 644 p.
Labit A., Chaland K., 2012. L’habitat groupé autogéré en France et en Allemagne : perspectives d'avenir dans le contexte du vieillissement démographique, Espace populations sociétés [En ligne], 2010/1, 2010, mis en ligne le $1^{\mathrm{er}}$ avril 2012, [http://eps.revues.org/3982].

Martouzet D. (dir.), 2014. Ville aimable, Tours, Presses universitaires François-Rabelais, coll. «Perspectives Villes et territoires », $384 \mathrm{p}$.

Membrado M., Rouyer A., 2013. Habiter et vieillir. Vers de nouvelles demeures, Toulouse, Erès, coll. « Pratiques du champ social », $278 \mathrm{p}$.

Nader B., 2011. Personnes âgées : les oubliées du territoire urbain?, Les déterminants socio-environnementaux de la santé des aînés, n 411, janvier-février, p. 29-30.

Nowik L., Thalineau A., 2014. Vieillir chez soi : les nouvelles formes du maintien à domicile, Rennes, PUR, coll. « Le Sens social », 222 p.

Pinet C., 2003. Vieillir aux États-Unis : Une géographie sociale et régionale des personnes âgées, Rennes, PUR, coll. " Géographie sociale », $253 \mathrm{p}$.

Sahmi N., Longé C., Bretas M., 2004. Concevoir un espace public accessible à tous : voirie, logements collectifs, ERP, IOP, lieux de travail, Paris, CSTB, 77 p. 